Revue

de l'histoire des religions

\section{Revue de l'histoire des religions}

$2 \mid 2012$

Élection, Loi et communauté : de l'Alliance à la cité chrétienne

\title{
Gratia Adami, gratia Christi. La nature, la Loi et la grâce dans le premier augustinisme
}

Gratia Adami, gratia Christi: Nature, Law and Grace in Early Augustinianism

Jérémy Delmulle

\section{(2) OpenEdition}

Journals

Édition électronique

URL : http://journals.openedition.org/rhr/7894

DOI : $10.4000 /$ rhr.7894

ISSN : 2105-2573

Éditeur

Armand Colin

Édition imprimée

Date de publication : 1 juin 2012

Pagination : 193-214

ISBN : 978-2200-92794-3

ISSN : 0035-1423

Référence électronique

Jérémy Delmulle, « Gratia Adami, gratia Christi. La nature, la Loi et la grâce dans le premier

augustinisme », Revue de l'histoire des religions [En ligne], 2 | 2012, mis en ligne le 01 juin 2015,

consulté le 04 mai 2019. URL : http://journals.openedition.org/rhr/7894 ; DOI : 10.4000/rhr.7894 


\section{Gratia Adami, gratia Christi La nature, la Loi et la grâce dans le premier augustinisme}

La question de la Loi, rapportée à la nature et à la grâce, fut l'une des principales préoccupations de ceux qui, après sa mort, défendirent Augustin contre les «semipélagiens», de Cassien à Fauste de Riez. Une analyse des probables filiations entre les textes d'Augustin et ceux de ses défenseurs, Prosper d'Aquitaine et Fulgence de Ruspe, permet d'observer les modalités et les motivations de l'émergence d'un augustinisme, entendu au sens d'un durcissement, conscient ou non, de la doctrine originelle. Si les termes et les développements restent en apparence identiques et profondément ancrés dans la théologie d'Augustin, leur application à des objets variés et leur insertion dans des contextes inédits contribuent à donner de la pensée d'Augustin une représentation biaisée.

\section{Gratia Adami, gratia Christi: Nature, Law and Grace in Early Augustinianism}

The question of the Law as related to nature and grace was one of the main concerns of those who, after Augustine's death, defended him against the so-called "Semipelagians", from Cassian to Faustus of Riez. An analysis of the probable filiations between the texts by Augustine and those of his defenders, Prosper of Aquitaine and Fulgentius of Ruspe, allows us to observe the terms and reasons for the emergence of Augustinianism, in the sense of a partly conscious and partly unconscious hardening of the original doctrine. Although the terms and developments are identical in appearance and deeply rooted in Augustine's theology, their application to various objects and their integration into new contexts help to give a distorted representation of Augustine's thought. 
Le testament dont il sera question est celui qu'Augustin a laissé ou, pour dire mieux, n'a pas laissé, malheureusement pour lui, à ses héritiers. La comparaison entre Augustin et Spinoza à laquelle se prête Milad Doueihi et l'intérêt marqué dont il fait preuve pour la notion de «relecture» invitent, en effet, à d'autres mises en perspective ${ }^{1}$. Il n'a pas toujours été loisible, en effet, de s'adonner à une telle confrontation directe de deux auteurs originaux, qu'on aurait pu lire dans le texte et dans l'absolu; et il n'est pas certain non plus qu'aujourd'hui nos lectures les plus objectives soient entièrement exemptes de tout conditionnement ou de tout apriorisme inconscients. Si l'on veut bien prendre en compte cet aspect, on ne peut lire l'histoire de la transmission des idées autrement que comme une chaîne d'intermédiaires, qui constituent fatalement autant d'obstacles et d'écrans. Car, pour que la doctrine d'Augustin se diffuse aussi rapidement et devienne très tôt sinon universellement reconnue, du moins incontournable, il a fallu qu'on fasse lire et relire, qu'on explique, qu'on commente même, pour un public peu avisé, les écrits du maître. L'un des premiers relais - celui, du moins, dont on ait conservé suffisamment d'œuvres pour en mesurer l'importance et l'influence avec assez d'exactitude - se trouve être un quasi contemporain d'Augustin, un Gaulois de Marseille, Prosper d'Aquitaine, à qui une étude célèbre de Dom Cappuyns a précisément attaché l'appellation de «premier représentant de l'augustinisme médiéval $»^{2}$.

On sait bien, pour reprendre de célèbres formules, que les mots en -isme ne sont que des bêtises, ou encore qu'ils empêchent de penser sérieusement. Mais force est de reconnaître que, dans le cas présent, le mot correspond particulièrement bien à la chose. Car l'augustinisme, tel qu'on l'entend habituellement - et en tout cas ici $^{3}-$ n'est pas celui de simples excerpteurs, comme Vincent de

1. Milad Doueihi, Solitude de l'incomparable. Augustin et Spinoza, Paris, Éditions du Seuil, 2009. Sur la «relecture», voir en particulier p. 15-24.

2. Maïeul Cappuyns, «Le premier représentant de l'augustinisme médiéval, Prosper d'Aquitaine», Recherches de théologie ancienne et médiévale, 1, 1929, p. 309-337.

3. Bien distinguer l'augustinisme théologique de l'augustinisme politique ou philosophique, dont les enjeux sont très différents. (On disposera bientôt d'une synthèse, concernant certes une période plus tardive: Eric Leland Saak, 
Lérins ou Eugippe, rassemblant quelques sententiae mémorisables pour rendre un compte fidèle de la pensée du docteur ${ }^{4}$; c'est celui d'hommes comme Prosper d'Aquitaine, ou surtout Fulgence de Ruspe qui, cent ans après Augustin, pousse jusqu'à un point extrême le rigorisme de certaines de ses positions. Or, il se trouve que ce «premier augustinisme», né très tôt (Augustin à peine mort), s'est immédiatement spécialisé dans un aspect seulement de la riche doctrine augustinienne: la question de la grâce et du libre arbitre, elle-même liée à celle de la prédestination; et c'est, qui plus est, à l'occasion d'une nouvelle controverse, issue de la polémique d'Augustin contre ce qu'on a défini comme le pélagianisme, que cette doctrine s'est développée 5 . Augustin, Prosper et Fulgence: c'est bien en effet la «triade» traditionnelle, la Sanctorum Patrum Trias, si prisée à Port-Royal ${ }^{6}$, censée fournir, sur les questions de la

Creating Augustine: Interpreting Augustine and Augustinianism in the Later Middle Ages, Oxford, Oxford University Press, à paraître en 2012.) Sur ces questions terminologiques, on peut se reporter à Salvatore Pricoco, «Una nota sull'agostinismo», in Filosofia, scienza, cultura. Studi in onore di Corrado Dollo, éd. G. Bentivegna, S. Burgio, G. Magnano San Lio, Soveria Mannelli, Rubbettino, 2002 (Biblioteca di studi filosofici; 17), p. 751-766.

4. Voir Vincent de Lérins, Excerpta (éd. R. Demeulenaere, Turnhout, Corpus Christianorum, series Latina [désormais CCSL] 64, 1985, p. 197-231), Eugippe, Excerpta ex operibus s. Augustini (éd. P. Knöll, Vienne, Corpus scriptorum ecclesiasticorum Latinorum [désormais CSEL] 9.1, 1885) ou même Prosper lui-même, dans son Liber sententiarum (éd. M. Gastaldo, CCSL 68A, 1972). À noter cependant que la littérature d'extraits est elle-même loin de présenter la garantie d'une stricte fidélité et ne manque pas, souvent, d'opérer nombre de travestissements : voir à ce propos Joseph T. Lienhard, «The Earliest Florilegia of Augustine», Augustinian Studies, 8, 1977, p. 21-31, en particulier p. 21: «To collect and publish excerpts from a man's writings may be a way of honoring him; but it is not always a way of doing him service» (voir aussi p. 25 et p. 31).

5. Il s'agit de ce qu'on nommera la «controverse augustinienne», connue encore souvent sous le nom de «semipélagianisme». Voir, à ce sujet, les synthèses récentes de Rebecca Harden Weaver, Divine Grace and Human Agency. A Study of the Semi-Pelagian Controversy, Macon (GA), Mercer University Press (Patristic Monograph Series ; 15), 1998; Donato Ogliari, Gratia et certamen. The Relationship Between Grace and Free Will in the Discussion of Augustine with the So-Called Semipelagians, Leuven - Paris - Dudley (MA), Peeters (Bibliotheca Ephemeridum Theologicarum Lovaniensium; 169), 2003 ; et Alexander Y. Hwang, Intrepid Lover of Perfect Grace. The Life and Thought of Prosper of Aquitaine, Washington, The Catholic University of America Press, 2009 (en particulier, p. 2-10; c'est lui qui propose la dénomination «controverse augustinienne» [p. 4]).

6. Le recueil, publié pseudonymement par [Jean Sinnich], Sanctorum patrum de gratia Christi et libero arbitrio dimicantium trias, Augustinus Hipponensis adversus Pelagium, Prosper Aquitanicus adversus Cassianum, Fulgentius Ruspensis adversus Faustum. Quorum propria verba sine ullo additamento 
grâce et du libre arbitre, un enseignement unifié et cohérent, à tout le moins complet. Mais loin d'être de purs citateurs, ces disciples sont eux-mêmes des théologiens qui, ayant lu Augustin, se sont chargés de diffuser sa pensée - et la diffusion s'opère rarement sans simplification réductrice ou sans déperdition - et même, vu qu'ils ont eu à le faire dans un contexte polémique, assez semblable pourtant à celui qui avait présidé aux prises de position d'Augustin, se sont vus contraints d'adapter ce discours à des problèmes en partie nouveaux, et donc à faire évoluer cette même doctrine, dont ils continuaient pourtant d'attribuer l'entière paternité à l'évêque d'Hippone.

En résumé, bien que initialement des récepteurs et des transmetteurs, les «augustiniens» sont finalement surtout des façonneurs. Étienne Gilson avait d'ailleurs très bien exprimé la difficulté ou le trouble que venait mettre ce filtre de l'augustinisme (au sens large et pluriel) dans notre appréhension d'Augustin luimême:

«On ne peut lire saint Augustin sans éprouver l'impression de l'immense importance de sa doctrine ni sans hésiter fréquemment sur la manière de l'interpréter. Tant de choses en sont nées qu'on craint de lui attribuer ce qu'elle a préparé sans le contenir et, inversement, de lui refuser ce qu'elle devait bien contenir au moins en germe, puisqu'elle lui a donné naissance » ${ }^{7}$.

Mais il est évident que la pensée d'Augustin est plus complexe, et que ses épigones sont, selon la règle, plus augustiniens qu'Augustin. S'attacher à observer la naissance de l'augustinisme, c'est donc se donner la possibilité de comprendre comment, en partie sciemment, en partie aussi malgré eux, des admirateurs d'Augustin ont créé à partir d'un matériau très vaste une doctrine qui fût plus facilement assimilable, au risque d'être, au moins partiellement, fausse.

summa fide referuntur, collectore Paulo Erinacho, [s.1.], [s.n.], 1648, a, en effet, sans doute bénéficié de la collaboration d'Antoine Arnauld, à qui seraient dus les titres et les sommaires (Philippe Sellier, Pascal et saint Augustin, Paris, A. Colin, 1970, p. 13 et n. 3), et a eu une influence considérable sur le Pascal des Écrits sur la grâce, qui tire de cet ouvrage la majorité des passages qu'il convoque et dont il donne une traduction, en s'en référant même explicitement (Euvres complètes, éd. Michel Le Guern, Paris, Gallimard [«Bibliothèque de la Pléiade»], 1998, t. 2, p. 209-316 et, pour les références précises, les notes concernées).

7. Étienne Gilson, Les métamorphoses de la cité de Dieu, Paris, J. Vrin, 2005 [1 $1^{\text {re }}$ éd. : 1952], p. 74. 
C'est cette toute simple idée que je voudrais développer, en prenant comme point de départ, mais pour le quitter très vite, l'un des premiers objets de l'ouvrage de Milad Doueihi: la question de la ou des lois, qui seule rend possibles les comparaisons auxquelles il se livre ensuite ${ }^{8}$.

\section{Gratia Adami Contre Gratia Christi: un développement POST-AUGUSTINIEN}

Le plus commode serait peut-être de partir d'une distinction qu'expose Augustin dans son Contre Fauste, écrit contre les manichéens bien avant que n'éclate la controverse avec Pélage.

«Il existe, écrit-il, trois genres de lois. Le premier c'est la loi des Hébreux, que Paul appelle [loi] "de péché et de mort"; le deuxième la loi des nations (gentium), qu'il appelle "naturelle"; et le troisième genre de loi c'est la vérité [comprendre: la loi chrétienne, le Christ étant la Vérité]»?.

Pour étudier la relecture/réécriture d'Augustin par ses premiers disciples, je délaisserai la dichotomie qui existe entre le premier et le troisième genre de loi (loi judaïque et loi chrétienne), dont traite Solitude de l'incomparable, pour m'intéresser à une autre opposition, qui n'est d'ailleurs pas entièrement étrangère à la première. C'est l'histoire doctrinale qui y oblige, puisque dans les controverses sur la grâce, que ce soit la querelle pélagienne ou la querelle augustinienne, le judaïsme entre très peu, sinon pas du tout: il s'agit avant tout d'interroger le système complexe de la justification, pour déterminer quelle part en revient à Dieu et quelle part à l'homme. De ce schéma d'une loi tripartite, conservons donc uniquement les deux derniers termes, la loi naturelle et la loi chrétienne. À ces deux lois se rattachent grosso modo deux sortes de grâce: pour le dire encore avec les mots d'Augustin, on doit distinguer «la grâce par laquelle nous sommes hommes» - c'est la grâce reçue du Créateur par Adam (la gratia Adami) - et «la

8. M. Doueihi, Solitude..., p. 5-9, qui synthétise les différents enjeux de l'essai.

9. Augustin, Contra Faustum, 19, 2, éd. J. Zycha, CSEL 25.1, 1897, p. 497 : «Sunt autem legum genera tria: unum quidem Hebraeorum, quod peccati et mortis Paulus appellat; aliud uero gentium, quod naturale uocat [...]; tertium uero genus legis est ueritas $[\ldots] »$. 
grâce par laquelle nous sommes chrétiens", celle qu'apporte le Médiateur (la gratia Christi) ${ }^{10}$. Même si la seconde de ces grâces doit être assurément considérée comme plus puissante que l'autre, il ne s'agit évidemment pas d'anéantir la première. Une conciliation des deux n'est donc pas a priori inenvisageable, puisque Augustin en revendique même une entre grâce et nature, faisant remarquer dans ses Révisions qu'en rédigeant contre Pélage un Sur la nature et la grâce il a voulu écrire un livre afin de défendre la grâce, et non d'attaquer la nature ${ }^{11}$. La meilleure définition de la grâce serait donc même celle qui réussirait à reconnaître à chacun de ces acteurs le rôle qui lui est propre dans l'acquisition du salut. Pour en différencier clairement la fonction, Augustin désigne, dans le Sur la correction et la grâce, la grâce d'Adam comme un adiutorium sine quo non, et celle du Christ comme un adiutorium quo. Toute la différence réside donc dans le caractère simplement nécessaire ou bien nécessaire et suffisant de chacune de ces grâces ${ }^{12}$.

10. Voir, par exemple, la distinction faite dans Augustin, Sermo 26, 9, éd. C. Lambot, CCSL 41, 1961, p. 353-354: «Videte tamen, fratres mei, quomodo illam generalem gratiam praedicent, qua creatus est homo, qua homines sumus. Et utique et cum impiis homines sumus, sed non cum impiis christiani sumus. Hanc ergo gratiam qua christiani sumus, ipsam uolumus praedicent, ipsam uolumus agnoscant, ipsam uolumus, de qua dicit apostolus: Non irritam facio gratiam Dei. » [«Voyez cependant, mes chers frères, de quelle façon ils prêchent la grâce générale, par laquelle l'homme a été créé, par laquelle nous sommes hommes. De toutes façons, nous sommes hommes avec les impies, mais nous ne sommes pas chrétiens avec les impies. Cette grâce, donc, par laquelle nous sommes chrétiens, c'est celle-là que nous voulons qu'ils prêchent, c'est celle-là que nous voulons qu'ils reconnaissent, celle-là dont l'Apôtre dit : "Je ne rends pas inutile la grâce de Dieu."»]. Voir surtout, pour la comparaison entre la grâce reçue par Adam et celle du Christ, De correptione et gratia, 10, 26 - 13, 42, Patrologia Latina (désormais $P L$ ) 44, col. 931-942; tr. fr. J. Pintard, Paris, Bibliothèque Augustinienne (désormais BA) 24, 1962, p. 327-367.

11. Augustin, Retractationes, 2, 42, éd. A. Mutzenbecher, CCSL 57, 1974, p. 124: «Librum ergo quo huic respondi, defendens gratiam non contra naturam sed per quam natura liberatur et regitur, de natura et gratia nuncupaui», [tr. fr. G. Bardy, $B A 12$, p. 523 : «Le livre par lequel je lui ai répondu pour défendre la grâce et non pour attaquer la nature qui est délivrée et régie par la grâce est appelé De la nature et de la grâce»].

12. Certes, les mots sont d'Augustin, De correptione et gratia, 12, 34, PL 44, col. 936: «Itemque ipsa adiutoria distinguenda sunt: aliud est adiutorium sine quo aliquid non fit, et aliud est adiutorium quo aliquid fit», [tr. fr. J. Pintard, $B A$ 24, p. 345: "Il faut de même distinguer deux secours: l'un est le secours sans lequel une chose ne se fait pas, l'autre est le secours par lequel elle se fait»], mais quinze siècles de théologie les ont employés et interprétés de façons si diverses qu'il semble difficile de formuler une conclusion autre qu'aporétique. Pour une 
Ces quelques rappels me paraissaient essentiels pour une exacte évaluation de la polémique augustinienne. Car de ces précisions découlent tous les désaccords qui ont opposé, d'un côté les Provençaux - en particulier Cassien, puis, un demi-siècle plus tard, Fauste de Riez - et les défenseurs autoproclamés d'Augustin -Prosper, contre Cassien, et contre Fauste, Fulgence. Les Provençaux accusent Augustin d'avoir amoindri la puissance du libre arbitre à un point tel que celle-ci finissait par être complètement détruite. En tout cas - et c'est tout ce que nous pouvons dire, avec prudence Prosper les accuse devant Augustin d'accuser ainsi Augustin ${ }^{13}$. Bien sûr, il est tout à fait possible qu'Augustin ait été lu de cette façon dans les milieux monastiques de la Gaule méridionale, et d'autres suspicions de fatalisme pourraient d'ailleurs venir s'ajouter à son témoignage ${ }^{14}$. Mais Prosper cite comme exemple représentatif de cette réaction le cas de Jean Cassien, dont il se trouve que nous

synthèse, voir la note complémentaire de Jean-Marc Chéné et Athanase Sage, «Les deux économies de la grâce d'après le "De correptione et gratia" », in $B A 24$, p. 787-799.

13. Prosper, Epistula ad Augustinum = Augustin, Epist. 225, 3, éd. A. Goldbacher, CSEL 57, 1911, p. 458: «Remoueri itaque omnem industriam tolli que uirtutes, si dei constitutio humanas praeueniat uoluntates, et sub hoc praedestinationis nomine fatalem quandam induci necessitatem aut diuersarum naturarum dici dominum conditorem, si nemo aliud possit esse, quam factus sit. » [trad. fr. J.-M. Chéné, BA 24, p. 397: «Ainsi, parler d'une disposition divine qui prévient les volontés humaines, c'est détourner l'homme d'agir et supprimer les vertus, et sous le nom de prédestination introduire une sorte de fatalisme; ou bien c'est dire que le Seigneur a créé des natures d'espèces différentes, s'il est vrai que personne ne peut être autre qu'il n'a été fait.»].

14. Hilaire fait également allusion à cette conclusion que les Marseillais tirent de la distinction opérée par Augustin entre la grâce d'Adam et celle de tous les hommes de leur temps; voir son Epistula ad Augustinum = Augustin, Epist. 226, 6, éd. A. Goldbacher, CSEL 57, 1911, p. 475 : «His uerbis sanctitatis tuae mouentur, ut dicant quandam desperationem hominibus exhiberi. [...]», [trad. fr. J.-M. Chéné, $B A$ 24, p. 427 : «Ces paroles de votre Sainteté les émeuvent à ce point que, d'après eux, c'est là en quelque sorte offrir aux hommes le désespoir.»]. Prosper le redit dans son Epistula ad Rufinum, 3, 4, PL 51, col. 79 A-B : "Quidam nostrorum (quod de ipsis multum dolendum est) occultis, sed non incognitis susurrationibus obloquuntur [...], dicentes eum liberum arbitrium penitus submouere, et sub gratiae nomine necessitatem praedicare fatalem». [tr. fr. F. Vinel, dans «Une étape vers l'affirmation du salut universel: Prosper d'Aquitaine, Lettre à Rufin sur la grâce et le libre arbitre, introduction et traduction », Revue d'histoire ecclésiastique, 90, 3-4, 1995, p. 367-395, ici p. 381: «Certains des nôtres (ce dont il y a beaucoup lieu de s'affliger à leur sujet) le contredisent avec des murmures qu'ils tiennent cachés, mais qui ne nous sont pas inconnus [...], en disant qu'il ébranle profondément le libre arbitre et que sous le nom de grâce c'est une nécessité fatale qu'il prêche.»] 
avons conservé les œuvres. Or, la comparaison que permet de faire la lecture, notamment, de sa Treizième conférence «Sur la protection de Dieu» laisse apparaître au contraire de plus grandes similitudes avec la pensée d'Augustin que Prosper veut bien le dire. Comme Augustin, mais par une voie différente, Cassien veut proposer une conciliation de la liberté et de la grâce: il développe ainsi sa théorie de la coopération, dans laquelle il défend que la perfection ne peut être atteinte que par la conjonction de l'action divine et de l'action humaine ${ }^{15}$. Mais là où, pour Prosper, le bât blesse, c'est lorsque, tout en reconnaissant la nécessité de la grâce divine pour l'accomplissement, par exemple, d'une bonne action, Cassien soutient aussi que l'homme n'a quelquefois pas besoin de la grâce, non plus, cette fois, pour l'accomplissement, mais pour le commencement même de cette bonne action ou de toute bonne volonté16. Autrement dit, l'homme peut sans l'aide de Dieu vouloir le bien, en vertu d'une petite étincelle, qui lui resterait de sa nature prélapsaire ${ }^{17}$, mais il ne peut en aucun cas l'accomplir sans Dieu. Selon Prosper, une telle assertion - qui pourtant s'efforce de conserver une mesure et un parfait équilibre dans cette définition d'une coopération - se rapproche par trop dangereusement des idées pélagiennes et surtout ne correspond pas à la position d'Augustin, si bien qu'il la considère comme forcément erronée, suivant le schéma tout à fait illogique $\mathrm{A} \neq \mathrm{B}$, or $\mathrm{C} \neq \mathrm{B}$, donc $\mathrm{A}=\mathrm{C}$. Il est assez remarquable, par exemple, que Prosper, en analysant les réactions

15. Cassien, Conlatio 13, 3, 5, éd. M. Petschenig, CSEL 13, 1886, p. 364 : «Quibus manifeste colligitur non solum actuum, uerum etiam cogitationum bonarum ex Deo esse principium, qui nobis et initia sanctae uoluntatis inspirat et uirtutem atque oportunitatem eorum quae recte cupimus tribuit peragendi.» [trad. fr. E. Pichery, Sources Chrétiennes 54, Paris, 1958, p. 151: «La conclusion manifeste de tout ceci, c'est que le principe des actes bons, mais aussi des bonnes pensées, est en Dieu, qui nous inspire le commencement de la bonne volonté, et nous donne encore la force et le moment favorable, pour accomplir nos saints désirs. »] Sur la doctrine de la coopération développée dans cette conférence, voir les analyses de D. J. MacQueen, «John Cassian on Grace and Free Will. With Particular Reference to Institutio XII and Collatio XIII», Recherches de théologie ancienne et médiévale, 44, 1977, p. 5-28; D. Ogliari, «The Conciliation of Grace and Free Will. Cassian's Conlatio 13 Revisited», Augustiniana, 50, 2000, p. 141173 ; et, dernièrement, A. Y. Hwang, Intrepid Lover..., p. 147-151, qui en fait l'un des deux modèles de salut, à côté du «modèle alternatif».

16. Voir surtout Cassien, Conlatio 13, 8, 4, qui est le passage critiqué par Prosper, Contra collatorem, 2, 3 et 19 [2 $2^{\mathrm{e}}$ déf.] (textes cités infra, n. 43 et 45-46).

17. Cassien, Conlatio 13, 7, 1 (texte cité infra, n. 44). 
provençales aux écrits augustiniens, commette le contresens dont se gardait explicitement son maître à propos du Sur la nature et la grâce, puisqu'il divise les protagonistes en deux seules catégories possibles: d'un côté les défenseurs de la grâce, de l'autre les défenseurs du libre arbitre ${ }^{18}$. Affirmer la moindre capacité du libre arbitre revient, dans ce raisonnement simplificateur, à s'opposer à la grâce. Allant dans le même sens, on notera un fait, certes littéraire ou rhétorique, mais qui en dit long sur l'indéniable parti pris de nos augustiniens. La controverse provençale a donné lieu, chez eux, à deux œuvres polémiques majeures (les autres n'étant généralement que des opuscules d'une bien moindre ampleur ${ }^{19}$ ): le Livre contre un conférencier de Prosper et le Sur la vérité de la prédestination et de la grâce de Dieu de Fulgence. À ces deux traités, d'abord, c'est le mot gratia qui sert d'incipit ( «Gratiam Dei», chez Prosper; «Deo gratias», chez Fulgence, ce qui relève plutôt du jeu de mots), mais surtout les deux auteurs affichent très nettement dès la première phrase quelle est cette grâce qui est l'objet de leur écrit: chez Fulgence, c'est «la grâce par laquelle nous sommes sauvés » (donc, nécessairement celle du Christ) ${ }^{20}$; quant à Prosper, encore

18. Voir surtout Prosper d'Aquitaine, Contra collatorem, 3, 1, PL 51, col. $221 \mathrm{C}$ $-222 \mathrm{~A}$ : «Sed nec cum haereticis tibi, nec cum catholicis plena concordia est. Illi in omnibus iustis hominum operibus liberae uoluntatis tuentur exordia; nos bonarum cogitationum ex Deo semper credimus prodire principia; tu informe nescio quid tertium et utrique parti inconueniens reperisti, quo nec inimicorum consensum acquireres nec in nostrorum intelligentia permaneres.» [ «Mais tu n'es en pleine concorde ni avec les hérétiques, ni avec les catholiques. Ceux-là, c'est dans toutes les œuvres justes des hommes qu'ils défendent les débuts de la volonté libre; et nous, nous croyons que c'est de Dieu que sortent toujours les commencements des pensées bonnes. Toi, tu as trouvé je ne sais quelle troisième solution informe, qui ne convient à aucun des deux partis, sans obtenir le consentement des ennemis, ni conserver d'intelligence avec les nôtres. »].

19. Outre le Contra Faustum en sept livres de Fulgence, perdu, exception doit être faite de l'Ad Monimum (éd. J. Fraipont, CCSL 91, 1968, p. 1-64), entièrement consacré à la question, mais qui ne se présente pas comme un ouvrage à proprement parler polémique. (Voir Guillaume Gabriel Lapeyre, Saint Fulgence de Ruspe. Un évêque catholique africain sous la domination vandale, Essai historique, Paris, P. Lethielleux, 1929, p. 211-212.) Le De uocatione omnium gentium de Prosper n'a pas non plus été pris en considération: lui aussi se détache de toute atmosphère directement polémique, et les distances qui y sont prises avec la pensée d'Augustin en font également, du point de vue de la diffusion doctrinale, une œuvre à part.

20. Fulgence de Ruspe, De ueritate praedestinationis, 1, 1, éd. J. Fraipont, CCSL 91 A, 1968, p. 458: «Deo gratias ago, sancti fratres Iohannes presbyter et Veneri diacone, cuius ope tales estis, ut pro gratia qua saluamur magno spiritu et feruore certetis ». [ «Je rends grâces à Dieu, saints frères Jean, prêtre, et Vénérius, 
plus explicite, il reprend mot pour mot l'expression d'Augustin que j'évoquais : «la grâce par laquelle nous sommes chrétiens $»^{21}$. Plutôt que de reprendre la discussion davantage en amont, suffisamment en amont pour espérer trouver une solution qui satisfasse tout le monde, ces deux auteurs se placent donc d'entrée de jeu du côté de la gratia Christi, la gratia Adami, devenue pour eux un pur synonyme de natura et de libre arbitre, étant laissée comme l'apanage de leurs adversaires $^{22}$.

Peut-être entrevoit-on déjà le risque que peut courir une telle doctrine lorsque, pour plus de clarté, on choisit de l'utiliser en l'adaptant et en lui ajoutant un exclusivisme (en l'occurrence assez trompeur) qu'elle n'avait d' abord pas. Ainsi, on assiste à la formation d'un augustinisme, entendu ici dans le sens d'une simplification à l'excès, qui ne peut donc s'empêcher de trahir la pensée originale, ce qui, dans un domaine tel que la théologie, ne peut aboutir qu'à une radicalisation.

\section{INTERPRÉTATION OU RÉDUPLICATION? L'EXEMPLE DE RM 2, 14-16}

Mais arrêtons-nous plus longuement sur un cas précis, qui nous permette d'examiner étape par étape ce processus de rigidification d'une pensée ou d'une argumentation. Le mot de gentes qui, des trois sortes de lois distinguées par Augustin que j'ai indiquées plus haut, servait à définir la deuxième, et au-delà les individus qu'il désigne,

diacre: c'est grâce à son aide que vous êtes de nature à combattre, avec beaucoup de courage et d'ardeur, en faveur de la grâce par laquelle nous sommes sauvés »]. On dirait exactement la même chose du début de l'Ad Monimum $(1,1)$ : Gratias ago Domino... (éd. J. Fraipont, CCSL 91, 1968, p. 1).

21. Prosper d'Aquitaine, Contra collatorem, 1, 1, PL 51, col. 215 A : «Gratiam Dei, qua Christiani sumus, quidam dicere audent a sanctae memoriae Augustino episcopo non recte esse defensam » [«La grâce de Dieu, par laquelle nous sommes chrétiens, certains osent dire qu'elle n'a pas été correctement défendue par l'évêque Augustin, de sainte mémoire »].

22. Dans cette controverse, l'une des principales pierres d'achoppement aura été la confusion, le manque de définition précise des termes en examen: les augustiniens et leurs adversaires s' affrontent sur un même terrain, et en ayant recours aux mêmes mots, mais sans pour autant prendre conscience que ces derniers ne recouvrent pas exactement les mêmes réalités chez les uns et chez les autres. Voir la démonstration de Paul Mattei, «Le fantôme semi-pélagien. Lecture du traité $D e$ gratia de Fauste de Riez», Augustiniana, 60, 1-2, 2010, p. 87-117 (p. 106-113), concernant l'articulation de la grâce primitive et de la grâce du Christ. 
se trouve être précisément l'objet de vives discussions entre les tenants des deux doctrines opposées. C'est bien là, en effet, l'enjeu essentiel du débat qui oppose les Provençaux aux augustiniens, comme d'ailleurs de celui qui mettait face à face Augustin et les pélagiens: quel est le sort des peuples qui n'ont pas la foi, ceux qui, en définitive, ne sont dotés que de la gratia Adami? Parce qu'il ne faut pas isoler la controverse théologique du contexte ascéticomonastique qui l'a suscitée, cela revient à poser la question de savoir quelle est la part qui, dans la recherche que tout croyant - et, plus encore, tout moine - mène de la perfection, revient à sa nature propre (puisque en somme le cas des gentes, autrement dit celui de l'incroyant, de l'homme sans Dieu, représente mise à nue la part proprement naturelle dont dispose chacun, que celle-ci soit ou non complétée par le don supplémentaire de la grâce du Christ).

Pour analyser un peu clairement comment le discours d'Augustin a pu être remployé, même (et surtout) indûment, par ses continuateurs, j'aimerais me concentrer sur un seul passage scripturaire, fréquemment allégué à cette occasion, et comparer les interprétations qu'en ont données, au cours des deux controverses successives, les protagonistes de l'un et l'autre partis ${ }^{23}$. Il s'agit de trois versets de l'Épître aux Romains (2,14-16), la plupart du temps cités d'un bloc:

«Quand des nations (gentes), sans avoir de loi, font naturellement ce qu'ordonne la loi, ils se tiennent lieu de loi à eux-mêmes, eux qui n'ont pas de loi. Ils montrent que l'œuvre voulue par la loi est inscrite dans leur cœur; leur conscience en témoigne également ainsi que leurs jugements intérieurs qui tour à tour les accusent et les défendent. C'est ce qui paraîtra au jour où, selon mon Évangile, Dieu jugera par JésusChrist le comportement caché des hommes.»

23. Qu'on veuille bien ne pas considérer l'emploi de ces termes comme l'acceptation aveugle des faits tels qu'ils nous sont présentés par les initiateurs, sinon les créateurs, de cette supposée controverse. Les recherches récentes sur la question ont bien montré l'importance de la reconstruction a posteriori du combat polémique et sont allées parfois jusqu'à nier la notion même de controverse (synthèse avec bibliographie dans Conrad Leyser, «Semi-pélagianisme», trad. fr. C. Pasquet, in Saint Augustin, la Méditerranée et l'Europe (IV -XXI siècle), dir. A. D. Fitzgerald, M.-A. Vannier, Paris, Les Éditions du Cerf, 2005, p. 1308-1315 [p. 1309]). Ceux que l'on désignera commodément comme «partisans» sont donc les écrivains que la postérité a considérés comme «semipélagiens », et donc quasiment hérétiques et, de l'autre côté, leurs contradicteurs, qui l'ont censément emporté. 
Augustin cite trois fois ces versets ensemble ${ }^{24}$. Mais la première fois, dans son Sur le sermon du Seigneur sur la montagne, écrit vers 394, cette citation ne lui sert qu'à prouver que c'est bien Dieu qui parle dans la conscience humaine ${ }^{25}$.

Les deux autres occurrences sont autrement intéressantes, en ce qu'elles sont d'une part considérablement plus développées, mais surtout mises directement au service de la polémique antipélagienne. On les trouve d'abord dans le Sur l'esprit et la lettre, de 412, et dans le Contre Julien composé dix ans plus tard. Mais si Augustin s'intéresse à ces quelques versets en particulier, c'est parce que Pélage, dans sa Lettre à Démétriade, les avait lui-même intégrés à son argumentation, pour soutenir qu'il avait pu se trouver, dans la période allant d'Adam à Moïse, des hommes qui «avaient vécu saintement et avaient plu à Dieu $»^{26}$. Mais, suivant Augustin, si l'on admet que la sainteté leur a été accessible, la gratia Christi ne servirait de rien.

Julien d'Éclane, dans le passage qu' Augustin retranscrit au livre IV de son traité, semble avoir tenu le même discours que son maître: «À travers eux (les hommes des gentes), écrit Augustin, tu t'es efforcé de prouver que même des hommes étrangers à la foi du Christ ont pu posséder la vraie justice» (c'est-à-dire la perfection, donc la sainteté $)^{27}$. La réplique d'Augustin est la même dans les deux cas: les peuples mentionnés par l'Apôtre, qui peuvent faire naturaliter et sine lege ce que la Loi commande, ce sont évidemment les Grecs ${ }^{28}$,

24. Le point de départ de ces quelques considérations m'a été donné par les fiches du Fonds Anne-Marie La Bonnardière, conservé à l'Institut des «Sources chrétiennes» de Lyon.

25. Augustin, De sermone Domini in monte, 2, 9, 32, éd. A. Mutzenbecher, CCSL 35, 1967, p. 122: "Quis enim scripsit in cordibus hominum naturalem legem nisi Deus? De qua lege apostolus dicit: Cum enim [...]».

26. Pélage, Epistula ad Demetriadem, 4, PL 30, col. 19 C: «Hac lege usi sunt omnes, quos inter Adam atque Moysen sancte uixisse atque placuisse Deo, Scriptura commemorat».

27. Augustin, Contra Iulianum, 4, 23, PL 44, col. 750 : «Per hos enim probare conatus es, etiam alienos a fide Christi, ueram posse habere iustitiam, eo quod isti, teste Apostolo, naturaliter quae legis sunt faciunt.»

28. Augustin, De spiritu et littera, 26, 44, éd. K.F. Urba, J. Zycha, CSEL 60, 1913, p. 198: «Proinde non uidetur alios hic significasse sub nomine gentium quam eos quos nomine Graeci supra significabat, cum diceret: [...] Iste autem Graecus nomine gentium significatus est naturaliter quae legis sunt facientium et quae scriptum habent opus legis in cordibus suis, profecto ad euangelium pertinent gentes, quibus lex in cordibus scripta est ». [ «Il ne semble donc pas qu'il ait désigné ici, sous le nom de peuples, d'autres hommes que ceux qu'il désignait plus haut 
mais les Grecs qui sont parvenus à la connaissance de l'Évangile ${ }^{29}$. Il le redit autrement contre Julien: l'Apôtre parle, dans ce passage, de chrétiens; et s'ils sont dits agir sine lege, c'est par opposition aux Juifs qui, eux, avant de connaître le Christ, avaient déjà une Loi ${ }^{30}$; on aurait peut-être mieux dit, d'ailleurs, que ces Grecs - ces païens en général - sont venus au christianisme ex nulla lege. Quant à la nature, dont il faut bien expliquer la présence dans ce verset, on ne doit pas, toujours selon Augustin, lire naturaliter littéralement, puisqu'il ne peut s'agir que d'une nature «corrigée par la grâce de Dieu » ${ }^{31}$, ce qui est donc l'inverse de l'état de nature invoqué par Pélage.

Une fois le débat transporté en Gaule, le même passage se trouvera cité par Cassien, et Prosper en critiquera longuement l'interprétation, en en développant une autre, entièrement tributaire de celle d'Augustin. Mais une grande différence sépare l'exégèse de Cassien de l'exégèse pélagienne: si Cassien a recours à cette citation, c'est avant tout pour prouver que la prévarication a fait apparaître chez Adam la science du mal (qu'il n'avait pas auparavant), sans pour autant lui faire perdre la science du bien (qu'il avait reçue lors de sa création); et de ce fait, lorsque l'Écriture dit que les Juifs ont été aveugles, ce n'est pas parce que leur nature ne leur donnait pas la connaissance du bien: il s'agissait d'une cécité non naturelle, mais volontaire ${ }^{32}$. Aussi les païens (ou les Gentils ou les nations)

du nom de Grecs, quand il disait: [...] Or, c'est ce Grec qui est désigné du nom de peuples qui font naturellement ce qui relève de la Loi et qui ont, écrite dans leur cœur, l'œuvre de la Loi; ils appartiennent donc à l'Évangile, ces peuples dans le cœur desquels la Loi a été écrite»].

29. Augustin, De spiritu et littera, 26, 46, éd. K.F. Urba, J. Zycha, CSEL 60, 1913, p. 200: «Si ergo gentes commemorans naturaliter quae legis sunt facientes et scriptum habentes opus legis in cordibus illos intellegi uoluit, qui credunt in Christum, quia non sicut Iudaei praemissa sibi lege ueniunt ad fidem,...». [ $\ll \mathrm{Si}$ donc, en faisant mention des peuples qui font naturellement ce qui relève de la Loi et qui ont, écrit dans leur cœur, l'œuvre de la Loi, il a voulu que l'on comprenne ceux qui croient au Christ, parce qu'ils ne viennent pas à la foi comme les Juifs, à qui la loi avait été déjà envoyée, $\ldots \gg]$.

30. Augustin, Contra Iulianum, 4, 25, PL 44, col. 750: «Ex qua fide placent, nisi ex fide Christi ? [...] Ideoque dicuntur sine lege naturaliter quae legis sunt facere, quia ex gentibus uenerunt ad Euangelium, non ex circumcisione, cui lex data est. »

31. Ibid., $P L 44$, col. 750 : «Et propterea naturaliter, quia ut crederent, ipsa in eis est per Dei gratiam correcta natura».

32. Cassien, Conlatio 13, 12, 3, éd. M. Petschenig, CSEL 13, 1886, p. 378-379: «Concepit ergo Adam post praeuaricationem quam non habuerat scientiam mali, boni uero quam acceperat scientiam non amisit. Denique non amisisse humanum 
possèdent-ils la possibilitas boni - nous verrons plus loin selon quelles modalités cette possibilitas peut devenir effective. C'est la seule façon possible de comprendre ce texte pour que soit sauve la relation entre grâce et libre arbitre: il ne faut pas, poursuit Cassien, attribuer tous les mérites des saints à Dieu de manière à n'attribuer à la nature humaine que ce qui est mal ${ }^{33}$. Pour être tout à fait honnête, avouons que Cassien n'est pas non plus, face à l'irréductible Prosper, le penseur innocent et impeccable. Car dans sa citation, au verset 16 , il «oublie» une partie de la phrase, qui n'est pas si négligeable: «au jour que Dieu jugera les secrets des hommes», écrit-il simplement, omettant à la fois «selon mon Évangile» et «par Jésus-Christ». Omission que l'on ne saurait expliquer par l'état du texte biblique dont il dispose - aucun autre témoignage ne permet de faire cette supposition ${ }^{34}$. C'est bien plutôt que, dans ce passage, la mention conjointe du Christ et de l'Évangile tendrait à valider l'hypothèse qu'on parle ici d'une nature pour ainsi dire christianisée, et non de la nature nue de l'homme issu d'Adam; il ne tardera pas, du reste, à faire intervenir la nécessité de la grâce du Christ. Mais cela n'a aucune incidence sur la réfutation de Prosper, qui ne relève même pas l'altération textuelle. Prosper reprend très fidèlement l'interprétation d'Augustin, en soulignant bien, grâce à un parallèle, l'opposition qui existe entre les chrétiens issus du judaïsme et ces autres, dont parle l'Apôtre, qui n'en viennent pas: selon ses termes, hérités de la distinction de Ga 5, 6, ceux-ci sont «appelés à partir du prépuce», comparativement aux premiers qui le sont à partir de la circoncision ${ }^{35}$. Prosper aussi, comme Augustin,

genus post praeuaricationem Adae scientiam boni etiam Apostoli sententia euidentissime declaratur, qua dicit : Cum enim [...]. Quo sensu etiam Dominus per prophetam increpat Iudaeorum non naturalem, sed uoluntariam caecitatem, quam ipsi sibi contumaciter inferebant.»

33. Cassien, Conlatio 13, 12, 5, éd. M. Petschenig, CSEL 13, 1886, p. 379380: «Vnde cauendum nobis est, ne ita ad Dominum omnia sanctorum merita referamus, ut nihil nisi id quod malum atque peruersum est humanae adscribamus naturae.»

34. Du moins, le fichier du Vetus Latina Institut de Beuron n'indique pas de manuscrit omettant cette finale; quant aux citations de ce verset chez d'autres auteurs, elles sont difficilement exploitables, puisque toute lacune pourrait tout aussi bien être volontaire.

35. Prosper d'Aquitaine, Contra collatorem, 10, 2, PL 51, col. 240 B: «Si de his Apostolus loquitur qui ex praeputio sunt uocati, et cum essent longe, facti sunt prope, credentes in eum qui, quorum aliquando non misertus est, nunc autem miseretur, et iustificans circumcisionem ex fide et praeputium per fidem, duos 
glose naturaliter en faisant valoir que seule est envisageable la restitution suivante: «naturellement [...], par une nature réparée et renouvelée $»^{36}$.

Comme, dans son De gratia, Fauste de Riez utilisera à son tour ces versets pour appuyer la thèse de la puissance de la nature humaine, Fulgence ne pourra faire l'économie d'une argumentation, qui ne sera en fait que la pure répétition de l'interprétation d'Augustin. Seulement, à cet endroit de son argumentation, Fauste se place sur un terrain sensiblement différent, dans la mesure où ce qu'il attribue à la nature que l'homme a reçue en Adam n'est pas la simple possibilité d'agir conformément à la loi ; il soutient que les hommes qui ont vécu avant le Christ avaient, en vertu de leur nature, jusqu'à la connaissance de Dieu (la notitia unius Dei) ${ }^{37}$. Il n'est pas nécessairement utile de reprendre l'explication de Fulgence, en tout point identique à celle d'Augustin et de Prosper: les nations de l'Épître aux Romains, explique-t-il, ne sont pas les incredulae gentes, mais bel et bien des fideles Christiani ${ }^{38}$.

condidit in semetipso, destructoque pariete inimicitiae Iudaeorum et gentium, in uno nouo homine fecit pacem, concludens omnia in incredulitate, ut promissio ex fide Iesu Christi daretur credentibus [...]». [«Si l’Apôtre parle des hommes appelés depuis le prépuce et qui, alors qu'ils étaient loin, se sont rapprochés, se mettant à croire en celui qui avait maintenant pitié de ceux dont il n'avait jadis pas eu pitié et, pour justifier la circoncision par la foi et le prépuce par la foi, a enfermé les deux en lui-même, rasé le mur d'inimitié entre Juifs et nations et réalisé la paix en un homme nouveau et unique, enfermant le tout dans l'incroyance, pour que la promesse soit donnée aux croyants par la foi en Jésus-Christ»].

36. Ibid., PL 51, col. 240 B-C: «ut legis plenitudinem et opera charitatis naturaliter exsequantur, reformata scilicet renouataque natura.»

37. Fauste de Riez, De gratia, 2, 10, éd. A. Engelbrecht, CSEL 21, 1891, p. 86: «In grandi profundo ignorantiae uolutantur qui dicunt, quod unius Dei notitiam habere non potuerint gentes ante Saluatoris aduentum». [ «Ils se vautrent dans un profond abîme d'ignorance, ceux qui disent que les peuples, avant la venue du Sauveur, n'ont pu avoir la connaissance du Dieu unique»].

38. Fulgence de Ruspe, Epistula 17, 48, éd. J. Fraipont, CCSL 91 A, 1968, p. 600: «Quem quidem Apostoli locum hi qui gratiae contradicunt gentibus incredulis assignare contendunt, ex hoc uidelicet quod etiam illi qui fidei gratiam non recipiunt illa quae ad honestatem morum et humanae societatis uinculum pertinent naturali quadam lege sic seruant [...]. » [«Ce passage de l'Apôtre, les contradicteurs de la grâce s'efforcent de l'appliquer aux peuples incrédules, de toute évidence parce que même ceux qui ne reçoivent pas la grâce de la foi conservent, grâce à une certaine loi naturelle, ce qui ressortit à la noblesse des mœurs et au lien de la société humaine [...].»] (Voir aussi Ep. 17, 49.) Pour l'identification avec les chrétiens, voir, plus loin, Ep. 17, 51, éd. J. Fraipont, CCSL 91 A, 1968, p. 603 : «Quod si quibusdam cognoscentibus Deum, nec tamen sicut Deum glorificantibus, cognitio illa nihil profuit ad salutem, quomodo hi poterunt iusti esse apud Deum, 
Cette confrontation montre assez bien, me semble-t-il, quelle destinée peut avoir un discours, censément irréfutable et sans réplique puisque, si l'on suit la logique de Prosper et Fulgence, il a été amplement développé par Augustin contre ses adversaires et que ces mêmes adversaires ont ensuite été officiellement condamnés par l'Église pour hérésie. Certes, dans cette interprétation des versets pauliniens deux partis se laissent délimiter: les uns (Pélage et Julien, Cassien et Fauste) voient dans les gentes l'homme sub lege constitutus, tandis que les autres (Augustin, Prosper et Fulgence) considèrent qu'il s'agit de l'homme sub gratia constitutus. Mais à aucun moment les augustiniens, d'une grande susceptibilité à l'égard des conséquences de la chute, ne s'avisent que les enjeux de la position provençale sont tout à fait différents de ce qu'il en était des thèses pélagiennes; ils ne s'aperçoivent pas que les thèses de Cassien et de Fauste sont à leur façon une autre forme de réfutation de la doctrine pélagienne ${ }^{39}$. Et le fait brut leur paraissant le même - à savoir que leur avis diverge de celui d'Augustin -, ils se contentent de leur opposer la même réponse à l'identique.

Cette confrontation fait remarquer aussi à quel point certains mots, jugés dangereux en eux-mêmes ou par les conséquences fâcheuses que leur lecture ou leur interprétation peuvent provoquer, doivent, à des yeux augustiniens, être tout bonnement bannis d'un discours que pourtant ils ont fondé. Pour reprendre comme exemple le cas le plus frappant, le mot natura et ses dérivés comme naturalis, naturaliter, dont nous venons de voir une occurrence, parce qu'ils agissent comme un signal et se trouvent, trop facilement, mais directement associés aux thèses de Pélage, Célestius et Julien, ne manquent pas au moins d'attirer l'attention des polémistes soi-disant partisans de la grâce. Dans son Contre un conférencier, Prosper a sélectionné et cité les passages de la Conférence XIII de Cassien

qui sic in suis moribus atque operibus bonitatis aliquid seruant, ut hoc ad finem Christianae fidei caritatisque non referant?» [«Et si certains, qui ont connu Dieu, sans pour autant se glorifier comme Dieu, n'ont tiré de cette connaissance aucun bénéfice pour leur salut, comment pourront être justes auprès de Dieu des hommes qui conservent dans leurs mœurs et dans leurs œuvres quelque part de bonté, mais sans la rapporter à la finalité de la foi et de la charité chrétienne?»].

39. Voir, à propos de Cassien, les analyses toujours très fines d'Augustine M. C. Casiday, «Cassian Against Pelagianism», Studia monastica, 46, 2004, p. 1-27, développées dans le ch. 2 de son Tradition and Theology...: «Cassianus contra Pelagianos», p. 72-118. 
qu'il jugeait les plus décisifs et les a résumés en douze propositions, dont onze lui paraissent irrecevables, lorsqu'elles ne sont pas pour lui carrément hérétiques. On compte dans cette Conférence de Cassien à peine neuf occurrences de mots de la famille de natura, réparties dans quatre passages de l'œuvre ${ }^{40}$. Il va sans dire que chacun de ces passages est regardé à la loupe par Prosper et finit même par être mis à l'index dans sa récapitulation finale ${ }^{41}$. Car évidemment, si l'adverbe naturaliter peut être polysémique et se rapporter, s'agissant de la citation de Paul, non à l'état de nature luimême, mais, comme nous l'avons vu, à l'état de grâce, lorsqu'il est utilisé par Cassien, en revanche, il ne peut avoir qu'une acception, évidemment en mauvaise part.

\section{LA VOIE NATURELLE DE L'AUGUSTINISME : VERS UN CONDITIONNEMENT NORMATIF}

Mais cette tendance excède assez généralement la simple mésinterprétation, souvent volontaire. Nous avons remarqué déjà aussi, avec l'exégèse de Rm 2, 14-1642, quel tour de force avait permis à Prosper d'asséner péremptoirement, avec la grande autorité d'Augustin, sa réponse à une question que Cassien ne lui posait même pas. Mais nous pourrions très bien aller jusqu'à dire que la lecture et la fréquentation d'Augustin ont entièrement façonné, et insensiblement, une perception nouvelle des choses. Par une sorte de réflexe conditionné, Prosper ne peut s'empêcher d'appliquer sur

40. Sur un total de 171 occurrences dans l'ensemble des Conférences (d'après le Thesaurus Iohannis Cassiani. Series A - Formae, cur. Cetedoc, Turnhout, Brepols, 1992, p. 121). Jean Cassien, Collatio 13, 9, 5; 12, 3 (bis) ; 12, 4; 12, 5 (bis) ; 12, 6; 12, 7; 17, 4 (éd. M. Petschenig, CSEL 13, 1886, p. 374, 378-380 et 394).

41. Voir Prosper, Contra collatorem, 4, 2 (= 19 [4e déf.]); 10, 1;11, 1;11, 2 (= 19 [8 $8^{\mathrm{e}}$ déf.]) $; 13,1$ (= 19 [9e déf.]); 14, 2 (PL 51, col. 224 A, 240 A, 242 B, 243 A, 247 B, 254 B, 267 A et 268 B-C).

42. On pourrait en dire autant - et on l'a fait - du célèbre «Dieu veut que tous les homme soient sauvés » $(1 \mathrm{Tm} 2,4)$ : pour les divers aspects de l'infléchissement imposé par les augustiniens, outre la bibliographie spécifiquement relative au $D e$ uocatione omnium gentium, voir A. Y. Hwang, «Augustine's Interpretations of 1 Tim. $2: 4$ in the Context of His Developing Views of Grace», Studia patristica, 43, 2006, p. 137-142; Roland J. Teske, «The Augustinianism of Prosper of Aquitaine Revisited», Studia patristica, 43, 2006, p. 491-503 (spécialement p. 496-503); A. Y. Hwang, Intrepid Lover..., surtout p. 208-220. 
le premier texte venu une grille de lecture augustinienne. Ainsi de ce qu'il fait, toujours contre Cassien, dans un dernier exemple. Le plus intéressant, dans le cas présent, c'est que l'opinion de Prosper évolue à l'intérieur même de son traité, chaque nouveau grief le délivrant, au fur et à mesure, de toute nécessité d'impartialité: hésitant au début, il est tout à fait catégorique à la fin, au moment de récapituler toutes ses positions et condamnations. Cassien pose donc plusieurs fois le problème des semina uirtutum, ces «semences de vertus » que Dieu a inséminées en Adam et qui auraient subsisté dans l'homme après la chute. C'est grâce à elles que tout être humain serait capable d'avoir l'initiative d'une bonne volonté ou d'une bonne action. Elles se manifestent en l'homme, dit Cassien, par une étincelle, qu'il suffirait de raviver. Prosper, qui ne voit partout dans l'homme naturel que de la cendre morte dont on ne peut plus rien tirer, refuse d'admettre la proposition suivante de Cassien:

«[Dieu] aperçoit-il en nous quelque commencement de bonne volonté, aussitôt il épanche sur nous sa lumière et sa force, il nous excite au salut, donnant la croissance au germe qu'il a semé lui-même ou qu'il voit sortir de terre par nos efforts $»^{43}$.

Quelques paragraphes plus haut, on lisait la même chose, mais exprimée par le biais d'une métaphore différente:

«Dès qu'il [Dieu] voit éclater en nous la plus petite étincelle de bonne volonté, ou qu'il la fait jaillir lui-même de la dure pierre de notre cœur, sa bonté en prend un soin attentif; il l'excite, il la fortifie par son inspiration $»^{44}$.

On ne peut mieux représenter la parfaite coopération entre le libre arbitre et la grâce: quelle que soit l'image choisie, c'est toujours la grâce de Dieu qui permet l'accomplissement de l'action ou de la pensée et qui mène à son achèvement le bien. Quant au commencement de ce même bien, il est attribuable soit à l'homme,

43. Cassien, Conlatio 13, 8, 4, éd. M. Petschenig, CSEL 13, 1886, p. 371 : «Qui cum in nobis ortum quendam bonae uoluntatis inspexerit, inluminat eam confestim atque confortat et incitat ad salutem, incrementum tribuens ei quam uel ipse plantauit uel nostro conatu uiderit emersisse» (trad. fr. E. Pichery, SC 54, 1958, p. 158).

44. Cassien, Conlatio 13, 7, 1, éd. M. Petschenig, CSEL 13, 1886, p. 369 : «Cuius benignitas cum bonae uoluntatis in nobis quantulamcumque scintillam emicuisse perspexerit uel quam ipse tamquam de dura silice nostri cordis excuderit, confouet eam et exsuscitat suaque inspiratione confortat », (trad. fr. E. Pichery, SC 54, 1958, p. 155). 
soit à Dieu; autrement dit, soit directement à la grâce divine (la gratia Christi), soit indirectement à elle, à travers la grâce initiale (la gratia Adami).

Dans le déroulement de sa réfutation, Prosper commence par reconnaître qu'une telle conception est, à la rigueur, défendable, dans la mesure où Cassien, écrit-il, «peut encore dire qu'il désigne la naissance de la bonne volonté, dont les commencements ont été plantés ou inspirés par Dieu» et que, finalement, à travers ces semences, on peut apercevoir le semeur, Dieu ${ }^{45}$. Mais à la fin de son ouvrage, où il passe brièvement en revue les passages de Cassien qu'il a examinés et résume le contenu de sa critique en une ou deux phrases, il ne retient qu'une chose: que cette assertion n'est pas catholique, étant donné que «ce qui avait été donné tout entier à la grâce est maintenant imputé en partie au libre arbitre $»^{46}$, comme si ce «libre arbitre» était différent de l'état dans lequel se trouvait l'homme disposant des semences qui, lorsque Prosper en avait fait mention pour la première fois, pouvaient légitimement renvoyer au Créateur. C'est-à-dire qu'il applique à un texte nouveau, non plus l'argumentation de son maître, mais le schéma simplifié qu'il a pu en dégager et qu'il a généralisé: dans un cas comme dans l'autre, distinguer aussi franchement dans les propos de Cassien la nature (même comme création de Dieu) et la grâce, ce n'est rien d'autre que de supposer que ce discours, voire tout discours, ne peut que se placer dans un cadre augustinien et qu'être lu en fonction de la position, jugée normale et vraie, de l'évêque d'Hippone. Il devient, certes, aisé de faire entrer Cassien dans des catégories prédéfinies, de le reformuler en termes augustiniens, pour mieux le réfuter en montrant qu'il n'obéit pas aux critères imposés a posteriori ${ }^{47}$. Mais c'est simplement que Cassien parle d'ailleurs; et une comparaison avec

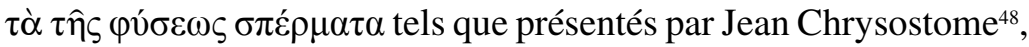

45. Prosper d'Aquitaine, Contra collatorem, 2, 3, PL 51, col. 220 A : «Et hic potest adhuc dicere illius se ortum bonae uoluntatis significasse, cuius a Deo sint plantata uel inspirata principia, quia ab illuminatis iam cordibus possint salubres prodire conatus, qui ideo ipsius hominis proprii esse dicantur, quia iam bonorum conatuum acceperit facultatem eorumque semina ad suum referantur auctorem ».

46. Prosper d'Aquitaine, Contra collatorem, 19, [2éf.], PL 51, col. 266 C: «Iam hic a praemissa definitione disceditur et quod totum gratiae datum fuerat ex parte nunc libero arbitrio deputatur».

47. Voir encore là-dessus A. M. C. Casiday, Tradition and Theology..., p. 27-29.

48. Voir, pour l'expression, Jean Chrysostome, In epistulam ad Ephesios

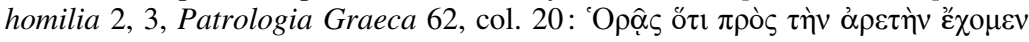


ou plus généralement avec toute la tradition théologique orientale, qu'on trouve chez un Évagre, par exemple, éclairerait bien mieux la conception de Cassien ${ }^{49}$. Prosper, par la force des choses «trop augustinien», se laisse ainsi lui-même tromper - ici et ailleurs ! -, et aura surtout considérablement trompé, pour de longs siècles, la quasi-totalité des lecteurs de Jean Cassien ${ }^{50}$.

Pour résumer - mais sans vouloir être à mon tour trop caricatural -, il faudrait faire remarquer que la doctrine d'Augustin touchant la grâce, qui l'avait emporté sur les pélagiens, s'est très rapidement diffusée, donnant lieu à une nouvelle controverse, née davantage encore, probablement, de la volonté de ses partisans qui ne souffraient aucune remise en cause de la pensée de leur maître. Pour la défendre - et peut-être surtout parce qu'il fallait la défendre - et la faire comprendre et adopter, aussi pour qu'elle ne puisse plus paraître trop ambiguë à certains (car, dans la controverse augustinienne, les Provençaux pouvaient se prévaloir d'une parfaite concordance entre leur doctrine et celle que l'évêque d'Hippone professait au début de son épiscopat, considérant son infléchissement final comme un durcissement aberrant ${ }^{51}$ ), des polémistes comme Prosper et Fulgence ont cherché à présenter la théologie augustinienne de la façon la plus

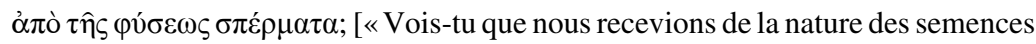
en vue de la vertu?»]. Le parallèle était déjà évoqué par Joseph Lombard, Jean Cassien. Sa vie, ses écrits, sa doctrine, Thèse présentée à la Faculté de Théologie protestante de Strasbourg, Strasbourg, 1863, p. 23, n. 2.

49. L'étude novatrice de Salvatore Marsili, Giovanni Cassiano ed Evagrio Pontico. Dottrina sulla carità e la contemplazione, Roma, Editrice Anselmiana («Studia Anselmiana», 5), 1936 doit maintenant être complétée et nuancée par les remarques d'A. M. C. Casiday, Tradition and Theology..., p. 5-6 et 139-160.

50. C'est précisément à cette influence du Contra collatorem sur la réception de la Collatio XIII de Cassien qu'est consacrée ma thèse de doctorat: «Prosper d'Aquitaine contre Jean Cassien. Édition critique, traduite et annotée du Contra collatorem et étude de sa réception» (Université Paris-Sorbonne). Pour un premier aperçu de la dépendance de la critique moderne vis-à-vis de Prosper, voir A. M. C. Casiday, «Cassian, Augustine, and De Incarnatione», Studia patristica, 38, 2001, p. 41-47 (surtout p. 41-44); Id., Tradition and Theology..., «Introduction», p. 6-10 et «Appendix I: Prosper's Influence on Modern Scholarship», p. 264-269.

51. Outre la mention de la difficile réception du De correptione et gratia en Provence (Prosper, Epistula ad Augustinum = Augustin, Epist. 225, 2), voir, entre autres, la remarque de Prosper, Contra collatorem, 21, 3, PL 51, col. 272 B : « ut scilicet quia in epistola papae, librorum pro quibus actum est, non expressus est titulus, hinc eo appareat non probatos, et istam in sanctum Augustinum laudationem pro anteriorum scriptorum meritis fuisse collatam» [«sous prétexte que, dans la lettre du pape, le titre des livres en question n'a pas été expressément indiqué, il 
cohérente possible, au point d'en faire un monolithe, puis, à force de n'avoir d'yeux que par elle, de l'imposer, aux autres comme à euxmêmes, comme la seule expression possible de la foi orthodoxe.

$\mathrm{Si}$, dans cet examen, j'ai accordé plus de place à Prosper qu'à Fulgence, c'est pour la simple raison que le Contre un conférencier de Prosper permet une confrontation plus directe avec le texte réfuté, la Conférence XIII de Cassien, et conduit donc à des réflexions un peu plus assurées que l'œuvre de Fulgence, dont le seul traité polémique sur la grâce, le Contre Fauste composé contre l'évêque de Riez, est perdu $^{52}$. Mais ce qui est vrai de Prosper l'est aussi de Fulgence, et la radicalisation qu'on peut observer dans l'augustinisme naissant va peut-être même s'accroissant au fil des décennies. Ainsi, c'est à Fulgence que l'on doit, par exemple, un compendium de la foi catholique (comprenez donc: de la foi augustinienne), le Sur la foi, à Pierre qui, après un exposé général destiné à embrasser l'ensemble des points de doctrine fondamentaux, en donne à lire et à retenir le résumé ramassé en quarante règles, selon des formules auxquelles leur concision ne permet plus d'être ambiguës ni même nuancées ${ }^{53}$. La formule «Hors de l'Église, point de salut» n'aurait sans doute pas eu une si grande postérité si elle n'avait été exprimée aussi nettement par ce théologien réputé le plus grand de son temps ${ }^{54}, a$ fortiori dans un traité que le Moyen Âge a pris l'habitude de lire et de copier comme étant d'Augustin ${ }^{55}$.

apparaîtrait par là qu'il ne les approuvait pas et que cette louange en faveur de saint Augustin ne lui a été conférée qu'en vertu des mérites de ses premiers écrits »].

52. Et il est impossible de rien connaître du contenu de cet ouvrage en sept livres (dont l'existence est attestée par le biographe de Fulgence, Ferrand de Carthage, la lettre 15 de l'épisolaire fulgentien ainsi que la notice du De uiris illustribus d'Isidore de Séville), célèbre pourtant par la publicité que lui a suscitée, au $\mathrm{XVII}^{\mathrm{e}}$ siècle, sa prétendue découverte par le faussaire Jérôme Vignier. Voir sur cette affaire Jean-Louis Quantin, "Combat doctrinal et chasse à l'inédit au $\mathrm{XVII}^{\mathrm{e}}$ siècle. Vignier, Quesnel et les sept livres contre Fauste de Fulgence de Ruspe», Revue des études augustiniennes, 44, 1998, p. 269-297.

53. Fulgence de Ruspe, De fide ad Petrum seu De regula fidei, éd. J. Fraipont, CCSL 91 A, 1968, p. 711-760; tr. fr. dans La règle de la foi, intr., trad., notes, guide thématique, glossaire et index d'O. Cosma, Paris, Migne («Les Pères dans la foi », 93), 2006 (avec bibliographie).

54. L'expression est de Bossuet, dans sa Défense de la Tradition et des Saints Pères, 1, 14: «S. Fulgence, le plus grand Théologien, et le plus saint Evêque de son temps » (Euvres de Bossuet, évêque de Meaux, revues sur les manuscrits originaux et les éditions les plus correctes, t. 5, Versailles, J. A. Lebel, 1815, p. 44).

55. Fulgence de Ruspe, De fide ad Petrum, 80-81 (= regulae 34-35), éd. J. Fraipont, CCSL 91 A, 1968, p. 757: «Firmissime tene et nullatenus dubites, 
Voilà donc, à partir de ces quelques exemples, comment semble se dessiner la première diffusion de la pensée d'Augustin: sa transmission au plus grand nombre, donc sa vulgarisation, n'a pu s'effectuer et jouir du succès qu'on lui connaît qu'au prix d'une détérioration assez notable. La pensée d'Augustin avait mis des décennies à se préciser, elle s'était améliorée à la faveur de telle ou telle controverse, de telle ou telle objection, elle consistait avant toute chose en des réponses circonscrites à des attaques ou à des questions. Et pour créer une vulgate augustinienne, on a voulu «systématiser» cette pensée. Systématiser n'a rien en soi de répréhensible, mais n'oublions pas la distinction qu'énonce à ce propos notre Littré: «L'esprit de système est la disposition à prendre des idées imaginées pour des notions prouvées. L'esprit systématique est la disposition à concevoir des vues d'ensemble. L'un est un défaut, l'autre peut être une qualité ${ }^{56}$. Et il n'est pas sûr que l'esprit de nos «augustiniens » ait été le second... En d'autres termes, et pour en revenir à notre conception testamentaire du début, on peut douter qu'ils aient été les légataires les plus propres à faire fructifier dans tous ses possibles la richesse de la pensée d'Augustin.

jeremy.delmulle@gmail.com

omnem extra Ecclesiam catholicam baptizatum, participem fieri non posse uitae eternae, si ante finem uitae huius catholicae non fuerit redditus atque incorporatus Ecclesiae. [...] Firmissime tene et nullatenus dubites, non solum omnes paganos, sed et omnes Iudaeos et omnes haereticos atque schismaticos, qui extra Ecclesiam catholicam praesentem finiunt uitam, in ignem aeternum ituros, qui paratus est diabolo et angelis eius.» [tr. fr. O. Cosma, op. cit., p. 107-108: «Tiens pour très certain, sans en douter le moins du monde, que tout homme baptisé hors de l'Église catholique ne peut devenir partie prenante de la vie éternelle si, avant la fin de cette vie, il n'a pas été rendu et incorporé à l'Église catholique [...]. Tiens pour très certain, sans en douter le moins du monde, que non seulement tous les païens, mais aussi tous les Juifs, et tous les hérétiques et schismatiques, qui finissent leur présente vie hors de l'Église catholique iront dans le feu éternel qui a été apprêté pour le diable et les anges qui lui appartiennent.»] Pour la portée de la position extrême de Fulgence, qui radicalise, en même temps qu'il en élargit l'application, l'adage véhiculé surtout, après Origène, par Cyprien de Carthage, voir Bernard Sesboüé, "Hors de l'Église, pas de salut». Histoire d'une formule et problèmes d'interprétation, Paris, Desclée De Brouwer, 2004, p. 68-70 et 72-74.

56. Émile Littré, Dictionnaire de la langue française..., Paris, Librairie Hachette et C $\mathrm{C}^{\mathrm{ie}}, 1885$, t. 4, p. 2119. 